\title{
Differentiating measuring coils for pulsed currents in the $\mathrm{MA} / \mathrm{MHz}$ range
}

\author{
E. Boggascha) and R. Grub
}

CERN-European Organization for Nuclear Research, 1211 Geneva, Switzerland

(Received 13 October 1986; accepted for publication 22 April 1987)

The design and construction of a high-performance differentiating pick-up coil for a 500-kA/ $4-\mu$ s pulsed current is described. Based on the Rogowski principle, the coil was made by using the printed-circuit technique. The conl is $1 \mathrm{~mm}$ thick; inclusive of electrostatic shielding and can be reproduced from one master print at nearly any size. Measurements up to $5 \mathrm{MHz}$ showed no significant change of self-inductance. We believe that the coil is a versatile tool to measure and monitor current pulsed in the megampere/megahertz range.

\section{INTRODUCTION}

For the plasma lens project at $\mathrm{CERN},{ }^{1}$ a pulse generator ${ }^{2}$ was built to deliver peak current pulses up to $500 \mathrm{kA}$ in a repetitive mode at $0.5 \mathrm{~Hz}$ into a common $z$-pinch load. ${ }^{3}$ The pulse generator consists essentially of four capacitor banks which are discharged simultaneously by four high-current pseudospark switches. ${ }^{4-7}$ The stored energy is transferred via four strip lines to the centrally located plasma lens. The main characteristics of the current generator are listed in Table I. A simplified block diagram is shown in Fig. 1.

Control of the switching delay time to achieve exact parallel discharge of the four banks, as well as examination of the plasma behavior in the lens, is essential. As the plasma load presents the main inductance of the discharge circuit, plasma dynamics resulting in pinching or instabilities can be observed by the total current signal. This requires a precise high stability current monitoring system. ${ }^{8-10}$ The approximate equation describing the peak discharge current for the LRC current generator is given below:

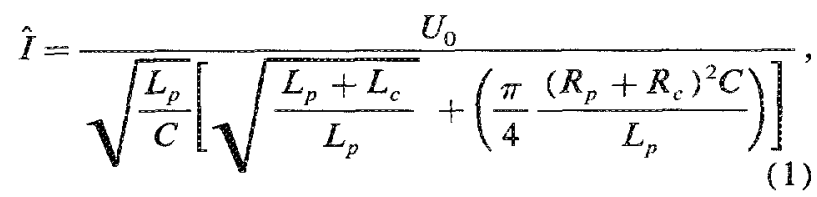

where $U_{0}$ is the charging voltage at $t_{0}, L_{p}$ is the inductance of

TABI.E 1. Characteristics of the pulse generator.

\begin{tabular}{|c|c|c|}
\hline 1. & Capacitance, total & $108 \mu F$ \\
\hline 2. & Nominal charging voltage & $16 \mathrm{kV}$ \\
\hline 3. & Maximum charging voltage & $20 \mathrm{kV}$ \\
\hline 4. & Stored energy at nominal voltage & $13.8 \mathrm{~kJ}$ \\
\hline 5. & Stored energy at maximum voltage & $21.6 \mathrm{~kJ}$ \\
\hline 6. & Peak discharge current at nominal voltage & $400 \mathrm{kA}$ \\
\hline 7. & Peak discharge current at maximum voltage & $500 \mathrm{kA}$ \\
\hline 8. & inductance of plasma lens & $20-100 \mathrm{nH}$ \\
\hline 9. & Circuit inductance & $10 \mathrm{nH}$ \\
\hline 10. & Circuit impedance & $9.6 \mathrm{~ms}$ \\
\hline 11. & Rise time to first current maximum & $1.6 \mu \mathrm{s}$ \\
\hline 12. & Equivalent half $n t$ half-sine-wave length & $7.4 \mu \mathrm{s}$ \\
\hline 13. & Initial $d I / d t$ at nominal current & $6 \times 10^{11} \mathrm{~A} \mathrm{~s}^{-1}$ \\
\hline 14. & Voltage reversal & $-45 \%$ \\
\hline 15. & Strip line cross section & $540 \times 11 \mathrm{~mm}$ \\
\hline 16. & Distance between conductors & $0.9 \mathrm{~mm}$ \\
\hline 17. & Repetition period & $2.4 \mathrm{~s}$ \\
\hline
\end{tabular}

the plasma, $L_{c}$ is the remaining inductance, $C$ is the capacitance of the capacitor banks, $R_{\mathrm{c}}$ is the circuit, and $R_{p}$ is the plasma resistance. The energy transfer efficiency from the capacitor banks to the plasma lens can be expressed as

$$
n=L_{p} \hat{I}^{2} / C U_{0}^{2} \text {. }
$$

Both $R_{c}$ and $L_{c}$ must, therefore, be kept as low as possible to minimize ohmic losses and to reach high peak currents; $\boldsymbol{R}_{c}$ and $L_{c}$ should, therefore, not be increased by the measuring system. ${ }^{11}$

This requirement excludes ohmic or inductive shunts ${ }^{12,13}$; measuring devices being both galvanically coupled to the circuit, and hence, requiring differential measuring at high potential. Hall-effect arrangements allow only punctual measurement at the pick-up point; the expected unsymmetrical current density in the 580 -mm-wide strip lines would falsify the measurement. Other methods, such as Faraday rotation, ${ }^{14}$ require additional equipment.

Furthermore, a locally space-distributed current density $\mathbf{j}(r, \Phi)$ is expected in the plasma. Therefore, current measurement directly around the plasma volume is required. For these reasons, only pick-up coils could be used. The integrated induced voltage in such coils, created by the varying magnetic fiux, represents a real image of the current.

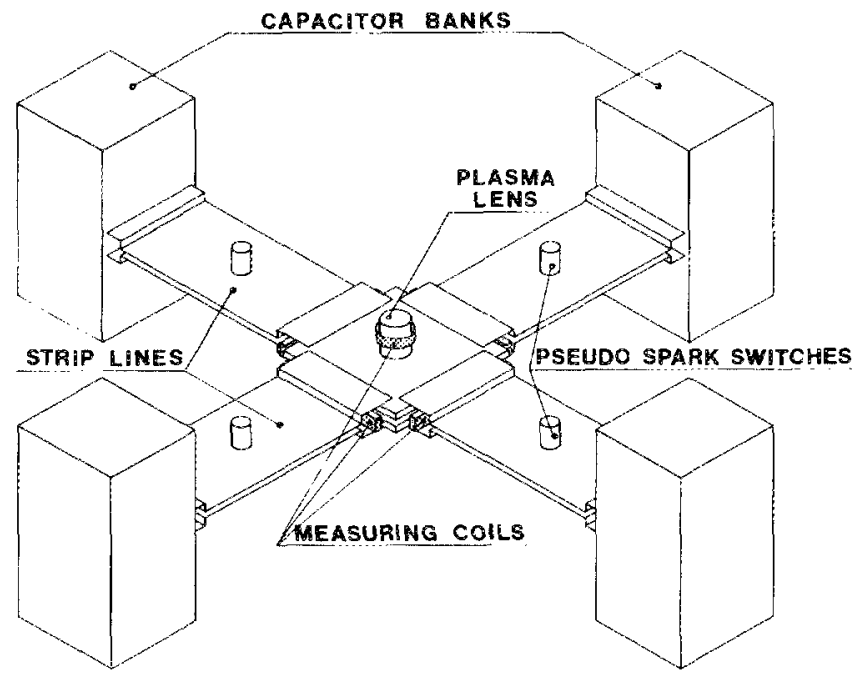

FiG. 1. Simplified block diagram of the pulse generator. 
These considerations led to the design and construction of the differentiating measuring coils described below.

\section{THEORY}

The Rogowski principle ${ }^{15,15}$ is shown in Fig. 2. For a distributed current density $j$ which is surrounded by a closed line circumscribing an area " $A$," neglecting displacement currents, Oersted's law is valid

$$
\oint_{i} \mathbf{H} \cdot d \mathbf{a}=\iint_{A} \mathrm{j} \cdot d \mathrm{~A} \text {. }
$$

Integration of the right-hand term leads to

$$
\oint_{a} \mathrm{~B} \cdot d a=\mu \mu_{0} I
$$

where $l$ is the cotal current penetrating " $A$." For a short line $f$, enclosing an area " $F$ " by surrounding " $a$ " the induction law can be expressed as follows:

$$
\oint_{f} \mathbf{E} \cdot d \mathbf{f}=-\frac{\partial}{\partial t} \iint_{F} \mathbf{B} \cdot d \mathbf{F} \text {. }
$$

The induced voltage signal $U_{i}$ is independent from the current distribution within the line " $a$ " only under the following conditions:

(a) Stray inductance, stray capacitance, and inter-turn leakage inductance can be neglected. This is admissible only for the application in our low-frequency domain.

(b) The pick-up coil is wound $n$ times around line a so that $F$ always has the same area for all turns and is perpendicular to a. For a finite number of $n$ turns, the integral in Eq. (5) can be written as a sum. The induced voltage then becomes

$$
U_{i}=F \cdot \sum_{v=1}^{n} \frac{d}{d t} B_{v \mid:},
$$

where $B_{\nu \|}$ is the field component parallel to the normal vector of $F$.

(c) The distance $\Delta d$ between turns is constant and small compared to magnetic field inhomogeneities along the integration line $a$. This with Eq. (4) leads to

$$
\Delta d \sum_{v=1}^{n} B_{v \|}=\mu \mu_{0} I
$$

For a coil length $l$ and uniform distances $\Delta d$, the relation is

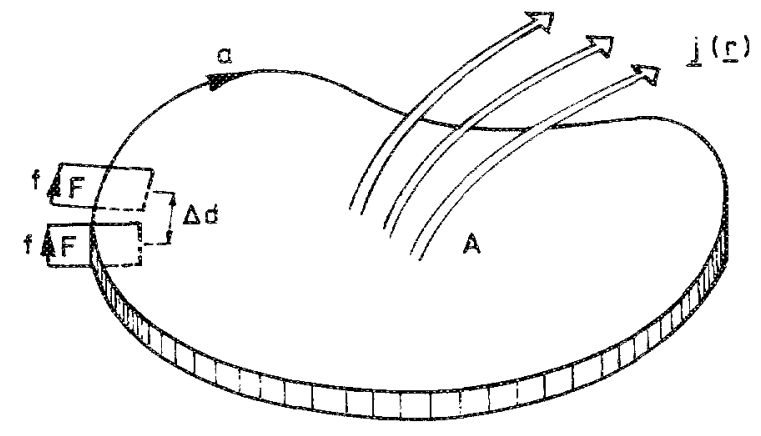

Fic. 2. Sketch of the Rogowsky principle.

$$
\Delta d=i / n
$$

Differentiating with respect to time in Eq. (7) and combined with Eq. (6) yieids the induced voltage

$$
U_{i}=\mu \mu \mu_{0} F \frac{n}{l} \frac{d l}{d t} .
$$

$U_{i}$ is now proportional to the time derivative of the current and the proportional factor

$$
M=\mu \mu_{0} F(n / l),
$$

contains only geometric parameters determining the sensitivity of the coil. The limit is reached when the current pulse length approaches the propagation time of the signal through the coil.

\section{I1. MEASURIRG CIRCURT}

The induced potential $U_{i}$ is transmitted by cables of impedance $Z$ to the monitor. Figure 3 shows the simplified circuit diagram. The symbols used in Fig. 3 are $U_{i}$ is the induced signal, $L_{c}$ is the coil inductance, $R_{c}$ is the resistance of the coil, $R$ is the terminating resistor, $Z$ is the cable impedance, and $U_{z}$ is the voltage on the terminating resistor.

Combining the circuit equation

$$
U_{i}=L_{c} \frac{d i}{d t}+\left(R_{c}+R\right) i
$$

with $U_{z}=R i$ yields

$$
U_{i}=\frac{L_{c}}{R} \frac{d U_{z}}{d t}+\left(\frac{R_{c}}{R}+1\right) U_{z} .
$$

As expressed by Eq. (9), $U_{i}$ is proportional to the time variation of the discharge current $d I / d t$. Therefore, two terms of the value of $\left(L_{c} / R\right)\left(d U_{z} / d t\right)$ should be considered:

$$
\frac{L_{c}}{R} \frac{d U_{z}}{d t} \geqslant\left(\frac{R_{c}}{R}+1\right) U_{z} .
$$

Here the measured signal $U_{z}$ is proportional to the discharge current $\hat{I}$. The coil is self-integrating with the time constant $\left(L_{c} / R\right)$.

The coils developed belong to a type, where

$$
\frac{L_{c}}{R} \frac{d U_{z}}{d t} \ll\left(\frac{R_{c}}{R}+1\right) U_{z} .
$$

At this condition, $U_{z}$ is proportional to $d I / d t$, the coil is differentiating.

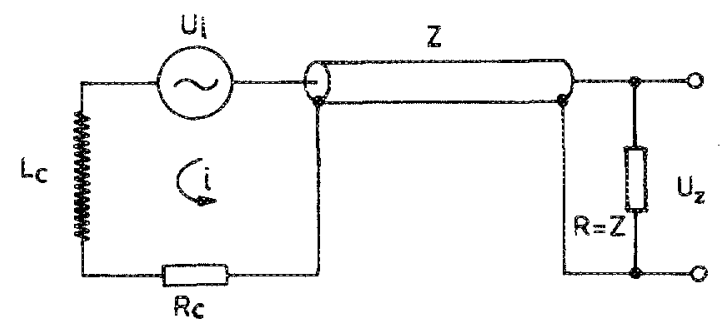

FIG. 3. Equivalent measuring circuit. 


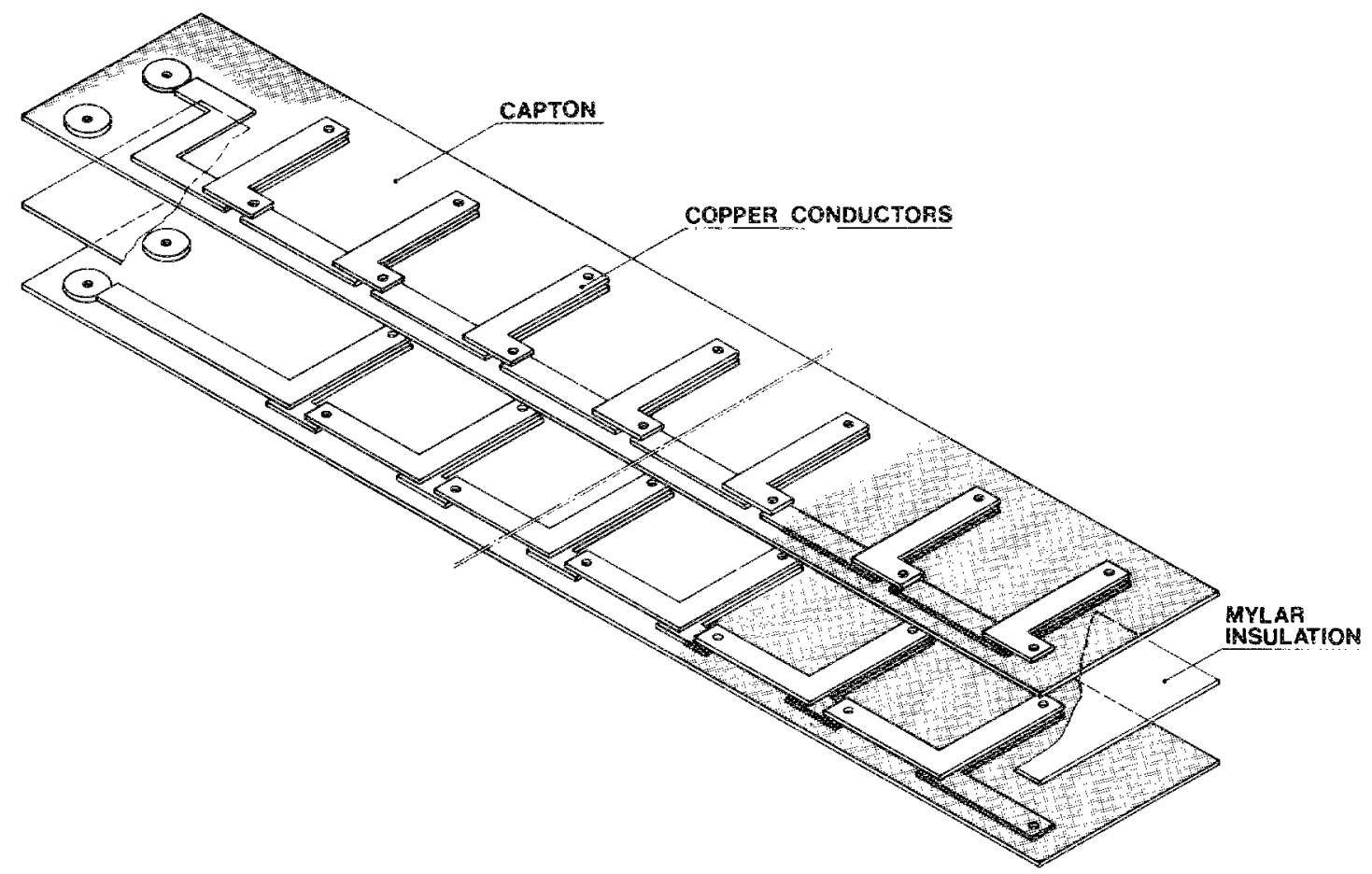

Fia. 4. The coil structure.

To evaluate Eq. (13), a harmonic approximation for $U_{z}$ of

$$
U_{z}=U_{z 0} \sin \omega t
$$

is assumed. The condition describing the upper limit for the induced frequency $\omega=\omega_{\mu l}$ of the described circuit, so that $U_{z}$ is still proportional to $(d I / d t)$, is

$$
L_{c}^{\left(\theta_{\mu l}\right.} \ll R_{c}+R .
$$

The resistance is mainly determined by the impedance of the cable between coil and preamplifier. Thus, the seifinductance of the coil must be minimized for high-frequency transmission.

Rearranging Eq. (15) gives

$$
L_{c} \ll\left(R_{c}+R\right) / \omega_{\mu l} \text {. }
$$

Inserting for $R_{c}+R \sim 100 \Omega$, a realistic vaiue for a shielded twisted pair of signal cables and $2 \pi \times 10^{6} \mathrm{~s}^{-1}$ for

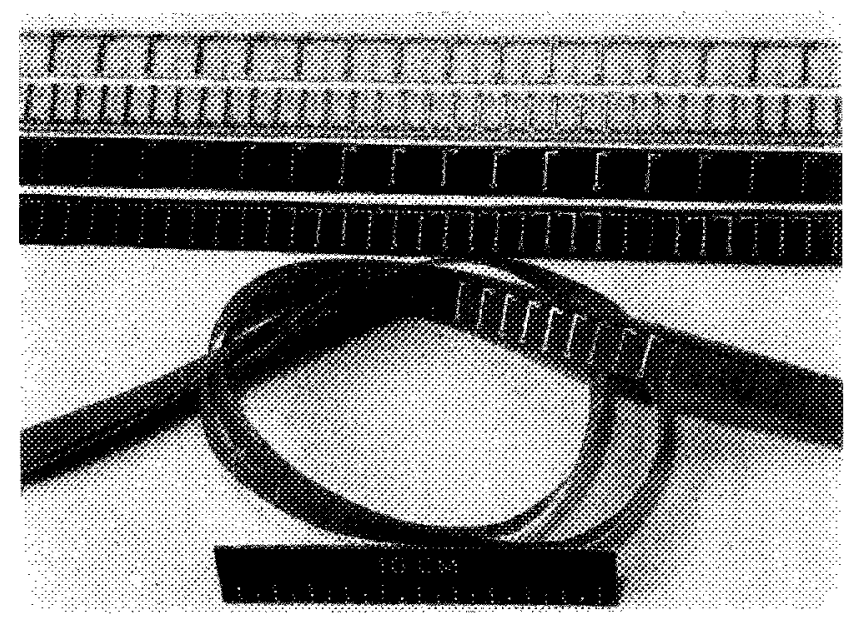

Fig. 5. Photograph of different coil types. $\omega_{\mu l}$, the maximal ringing frequency of the pulse generator, yields

$$
L_{c} \ll 1.6 \times 10^{-5} \mathrm{H} \text {. }
$$

This low inductance for a coil of $840 \mathrm{~mm}$ length, i.e., the circumference of the plasma cylinder, could be easily achieved by using the printed-circuit-board technique. The coils, twice $125 \mu \mathrm{m}$ thick, have the following features:

(a) Low inductance.

(b) Greatest flatness.

(c) This extreme flatness yields high mechanical flexibility.

(d) Either the $d l / d t$ and/or the integrated current signal can be measured.

(e) Excellent spatial integration over the current cistribution.

(f) Selection of the output voltage by varying the circuit board thickness.

(g) The four-layer technique (two coils superimposed) brings the terminals to the same side. Voltage signals induced by flux penetration into the measuring coil are, therefore, suppressed.

(h) Easy reproducibility at low tolerance.

(i) Superior long-term stability.

(j) Low cost.

These considerations lead to the development of the measuring coils described.

\section{CONSTRUCTION OF THE COILS}

Figure 4 shows a schematic view of the coil structure. The space available being only $20 \mathrm{~mm}$ between the strip lines and the central support of the plasma lens, limited the width of the coils, including the electrostatic shielding (Sec. IV). 
TABLE II. Main data of the coils.

\begin{tabular}{|c|c|c|c|c|c|c|}
\hline $\begin{array}{l}\text { Coil } \\
\text { No. }\end{array}$ & $\begin{array}{c}\text { Number } \\
\text { of tums } \\
\text { (n) }\end{array}$ & $\begin{array}{c}\text { Thickness } \\
(\mu \mathrm{m})\end{array}$ & $\begin{array}{l}\text { Length } \\
\text { (mm) }\end{array}$ & $\begin{array}{l}\text { Width } \\
\text { (mm) }\end{array}$ & $\begin{array}{l}\text { Winding } \\
\text { density } \\
(\mathrm{cm} / \mathrm{n})\end{array}$ & Use \\
\hline$b$ & $2 \times 38$ & $2 \times 125$ & $2 \times 570$ & 10 & 0.75 & Strip line \\
\hline $\mathrm{c}$ & $2 \times 76$ & $2 \times 500$ & $2 \times 570$ & 10 & 0.375 & Strip line \\
\hline$d$ & $2 \times 38$ & $2 \times 500$ & $2 \times 570$ & 10 & 0.75 & Strip line \\
\hline $\mathfrak{e}$ & $2 \times 114$ & $2 \times 125$ & $2 \times 840$ & 10 & 0.375 & Lens \\
\hline
\end{tabular}

This resulted in an effective width of the turns of $10 \mathrm{~mm}$. The distance of $10 \mathrm{~mm}$ between turns was selected to compensate for even small current displacements in the strip lines.

Introducing the necessary parameters in Eq. (9) for a selected output voltage of $100 \mathrm{~V}$ at a current rise of $5 \times 10^{11}$ A $\mathrm{s}^{-1}$ and $100 \mathrm{~cm}$ length it follows that

$$
F n \sim 1.6 \times 10^{-4} \mathrm{~m}^{2} \text {. }
$$

This yields a coil thickness of $160 \mu \mathrm{m}$ at $n=100$ turns. Based on these data, different types of coils represented in Fig. 5 were constructed. The nain parameters are compiled in Table II.

The winding density $l / n$ which determines the integration step for the spatially distributed current density was selected to be either 0.750 or $0.375 \mathrm{~cm} / \mathrm{n}$. The inductance $L$ of the coil depends only linearly and not quadratically on the length $l$, and therefore on $n$, and is determined by the strip. line geometry and can be expressed as

$$
L / l=\mu_{0}(d / w) \text {. }
$$

\section{SHIELDING}

Time dependent electrical fields are present in the vicinity of the strip lines and plasma discharge and induce superimposed perturbation signals. Special care must be taken to minimize the undesired noise signals for both the strip-line coils and the plasma bobbin by using appropriate shielding.

Space restriction round the plasma lens allowed only a sandwich-type arrangement as shown in Fig. 6.

It is obvious that the thickness of the polyester film represents a compromise between minimum capacitive coupling and maximum shielding effect. It may be worth mentioning that the earthing of the copper layers is crucial.

The space available for the strip-line coils allowed to house a closed M-U shape shielding is shown in Fig. 7.

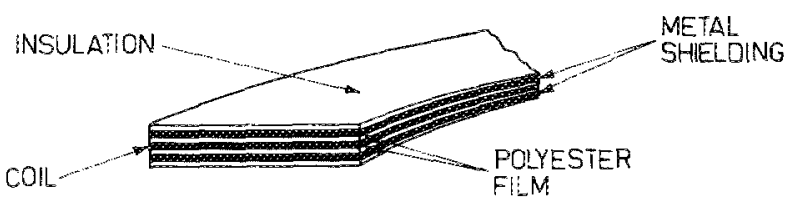

FIG. 6. Layout of an electrostatic shielding.
Several coils are accommodated in the metallic, nonmagnetic case, permitting simultaneous $d I / d t$ and $\hat{I}$ observations as well as interlock connections. The efficiency of this type of shielding is shown in Fig. 8.

\section{RESULTS AND MEASUREMENTS}

The results of the inductance measurements up to 5 $\mathrm{MHz}$ are shown in Fig. 9. All coils tested have values of less than $1000 \mathrm{nH}$ and no resonant frequency was detected. Coil $d$ was selected for the current measurements of the four strip lines and coil $f$ was taken to monitor the current in the plasma lens.

Equation (15) gives the limit for the upper frequency $\omega_{\mu l}$, where the induced voltage $U_{i}$ is still proportional to the rate of change of the current $d l / d t$. The measured inductance $L \sim 5 \times 10^{-7}$ and $7 \times 10^{-7} \mathrm{H}$ for coils $\mathrm{d}$ and $\mathrm{f}$, respectively, is more than a factor of 20 lower than stipulated in Eq. (17).

The magnetic stray field outside the strip line proved to be smaller by a factor of 40 compared to the inner field. Therefore, the measuring coils were inserted only between the plates, thus not surrounding the conductor.

Two sandwich-type shielded coils (f) surrounded the lens leading to a simuitaneous $\hat{I}$ and $d I / d t$ signai. An example of current signals is depicted in Fig. 10.

Calibration ${ }^{17}$ up to $80 \mathrm{kA}$ against a high precision, low inductive shunt shows excellent linearity and a realistic re-

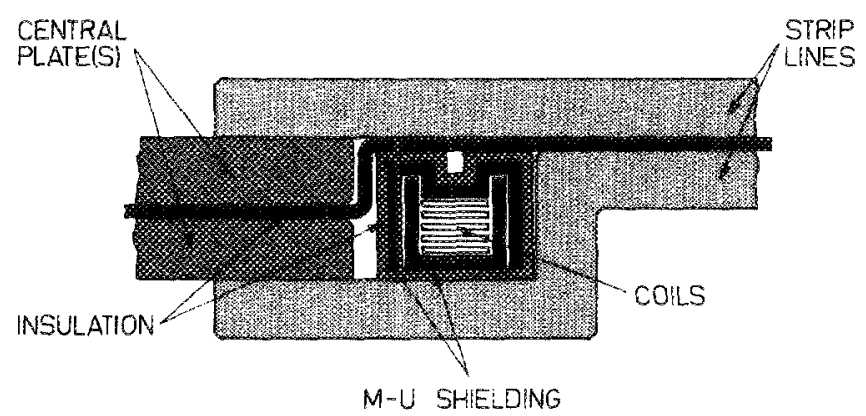

Fig. 7. Transverse cross section of coils and M-U-type shielding near central plate. 


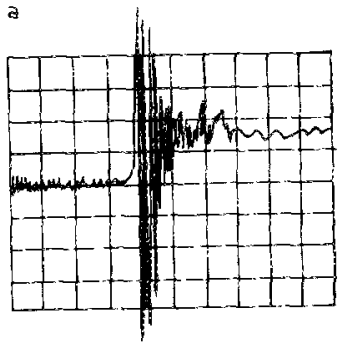

$\mathrm{di} / \mathrm{dt} ; 5 \mathrm{~V}-100 \mathrm{~ns} / \mathrm{div}$

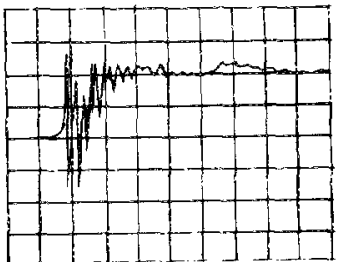

$d / / d t ; 5 v-100 \mathrm{~ns} / \mathrm{div}$

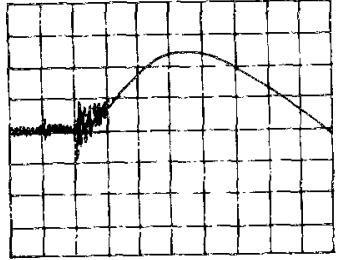

f: $10 \mathrm{kA}-1 \mu 5 / \mathrm{div}$

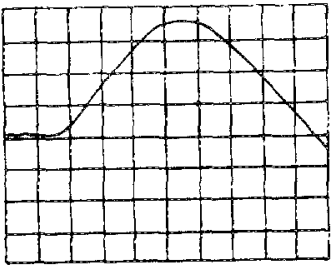

$\tilde{\mathrm{I}} ; 7,2 \mathrm{kA}=1 \mu \mathrm{s} / \mathrm{div}$.

Fig. 8. Effect of shielding on noise level for: (a) sandwich-type shielding and (b) M-U-type shielding.

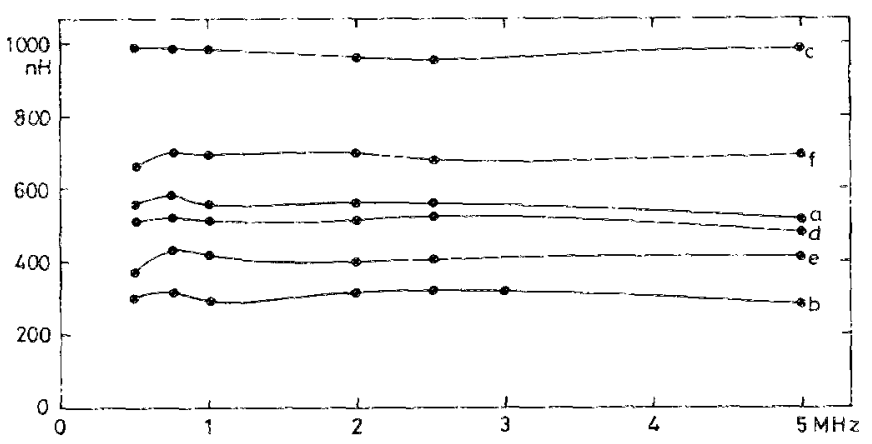

FiG. 9. Variation of self-inductance vs frequency.

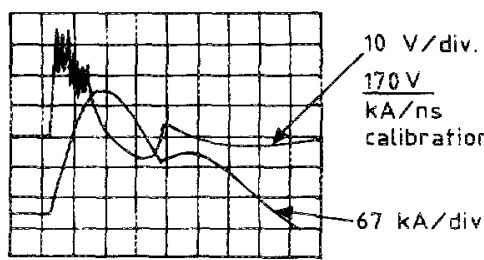

a 1 us/div.

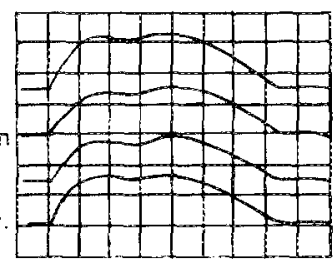

b $1 \mathrm{us/div.}$

FIG. 10. Current signals of a pinched discharge: (a) (dI/dt top) $)$ and $\hat{I}$; (b) simultaneous registration of the four strip-line currents $(4 \times 100 \mathrm{kA})$.

production of the pulse shape with a passive $1-\mathrm{ms} R C$ integration circuit. The ratio charging voltage/coil signal was found to be linear over the full range up to $400 \mathrm{kA}$.

\section{DISCUSSION}

The common printed-circuit-board techniques allow us to reproduce from one master print, Hexible multipurpose measuring coils of nearly any size.

For current pulses of higher frequencies, as in the application described, the self-inductance of the coils can be lowered by reducing the printed-circuit thickness. In addition, Eq. (9) shows that the increased $d I / d t$ values associated with higher frequencies require a smaller geometric factor $M$, to reach a comparabie signal amplitude. Thus, the inductance can be further reduced.

\section{ACKNOWLEDGMENTS}

The authors wish to thank their colleages $\mathrm{H}$. Riege and $V$. Brückner for helpful conversations. This work was performed as part of CERN's ACOL Project under the leadership of E. Jones from the PS Division. The encouragement of P. Lazeyras of the Experimental Physics Facilities Division is also gratefully acknowledged.

a) Permanent address: Physikalisches Institut der Universität ErlangenNürnberg, D-8520, Erlangen, Germany.

'L. DeMenna, G. Miano, B. Autin, E. Boggasch, K. Frank, and H. Riege, in Proceedings of the IVth National Congress of Quantum Electronics and Plasma Physics, Vol. 1 (1984), p. 273 and CERN/PS 84-13 (AA).

${ }^{2} \mathrm{E}$. Boggasch, H. Riege, and V. Brückner, in CERN/PS 85-30 (AA) and Proceedings of the 5th IEEE Pulsed Power Conference, Arlington, VA, p. III-36 (1985), pp. 820-823.

${ }^{3}$ C. A. Ekdahi, Rev. Sci. Instrum. 51, 1645 (1980).

'J. Christiansen and C. Schultheiss, Z. Phys. A 290, 35 (1979).

${ }^{5}$ D. Bloess, I. Kamber, H. Riege, G. Bittner, U. Brückner, C. Christiansen, K. Frank, W. Hartmann, N. Licser, C. Schultheiss, R. Seebock, and W. Steudtner, Nucl. Instrum. Methods 205, 173 (1983).

${ }^{6} \mathrm{G}$. Mechtersheimer, R. Kohler, T. Lasser, and R. Meyer, J. Phys. E 19, 466 (1986).

${ }^{7}$ E. Boggasch and H. Riege, in Proceedings of the XVIth International Conference on Phenomena in Ionized Gases, Vol. 2 (1985), p. 567 and CERN/ PS 85-4 (AA).

${ }^{*}$ M. Anderson, Rev. Sci. Instrum. 42,915 (1971).

${ }^{9}$ D. G. Pellinen and P. W. Spence, Rev. Sci. Instrum. 42, 1699 (1971)

D. G. Pellinen, Rev. Sci. Instrum. 42, 667 (1971).

1 'M. S. Di Capua, Keynote paper presented at Workshop on Measurement of Electrical Quantities in Pulse Power Systenns, National Bureau of Standards, Boudder, CO, 2-4 March 1981.

${ }^{12}$ A. J. Schwab, High-Voltage Measurement Techniques (M.I.T., Cambridge, MA, 1972), pp. 163 191.

${ }^{13}$ D. Kind, An Introduction to Figh-Voltuge Experimental Technique, 1st ed. (Vicweg, Braunschweig, Germany, 1978), pp. 50 52.

${ }^{14}$ G. I. Chandler and F. C. Jahoda, Rev. Sei. Instrum. 56, Part II, 852 (1985).

${ }^{15}$ V. Nassisi and A. Luches, Rev. Sci. Instrum. 50, 900 (1979).

${ }^{16}$ D. G. Pellinen, M. S. Di Capua, S. E. Sampayan, H. Gerbracht, and M. Wang, Rev. Sci. Instrum. 51, 1535 (1980).

${ }^{i 7}$ A. I. Gerasinov and E. G. Dubinov, Instrum. Exp. Tech. 26, (2), Part 2, 466 (March-April 1983). 